\title{
ON THE APPLICATION OF FULLY ANALYTICAL SOLUTION FOR THE COMBINED-ORDER DESIGN PROBLEM OF FREE GEODETIC NETWORKS
}

\author{
Rahil A. M., El Gohary A. M., Doma M. I. \\ Civil Engineering Department, Faculty of Engineering, Shebin El-Kom \\ Minoufiya University, Egypt
}

\begin{abstract}
Free geodetic networks are still very popular in geodesy, surveying and mapping mainly due to their unique property of being independent of errors in external data. The free "floating" network is a network in which only the internal shape is given by measurements of relative nature (Schmitt, 1982). In such a case, the corrections solution vector to the approximate coordinates is derived by selecting the best coordinate system. In other words, the term free network refers to a network whose adjustment is made free from any kind of external constraints. This means that the network can freely translate, rotate or undergo scale change in space. Optimization means minimizing or maximizing an objective function which represents the criteria adopted to define the "quality of a network". Generally the quality of a control network is characterized by its precision, reliability, strength and economy (Seemkooei, 2001; Kaplan, 2004). In this paper we focus on designing an optimal network configuration and optimum observing plan (Combined-Order Design Problem) in the sense that they will satisfy the present network quality requirement at a minimum cost. A small free geodetic network are simulated and its optimal design is solved by nonlinear programming method. The obtained results are discussed and analyzed.
\end{abstract}

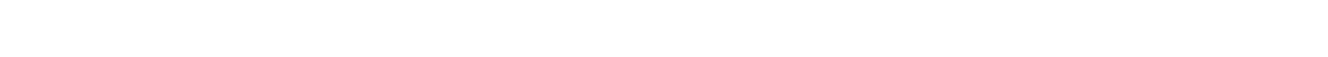

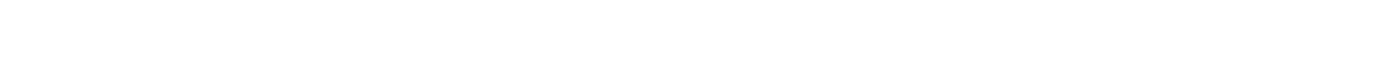

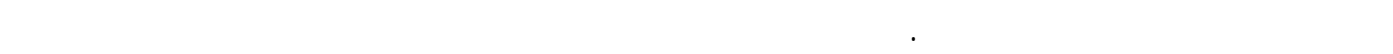

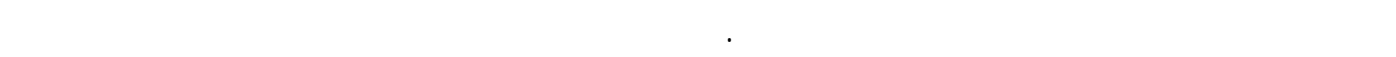

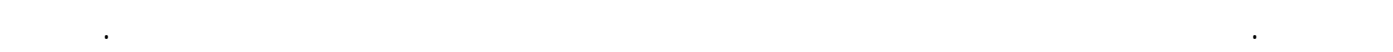

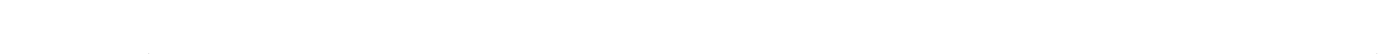

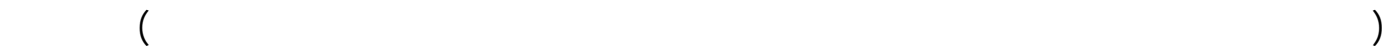

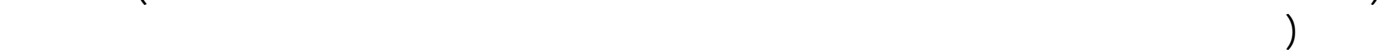
الأرصاد). وقد نجح هذا النموذج وبليتخدالم برنلمج التخطيط الفاعلي في تصميم وإيجاد القيم المثلى للمتغيريرات اللشبكت الحرة. وقد مق لستخدم البرمجة اللاخطية في جل النموذج الرياضي الفي المقترح.

\section{INTRODUCTION}

In general, the main task of optimal design of a geodetic network is comprised of:

口 Determination of the optimal distribution of network points

口 Selection of measurement techniques, and

口 Computation of the optimal distribution of the required observational precisions among heterogeneous observables.

In the past, it was very difficult, if not impossible, to solve for all aspects of network optimization in a single mathematical procedure. Instead, the problem of network design was divided into sub-problems in which some progress could be made. The accepted classification was; (Kunag, 1996; Kaplan, 2004; Rahil, 2005):
- Zero-Order Design (ZOD): the reference framework of network is defined;

- First-Order Design (FOD): the geometric shape of the network is defined, and the elements to be measured are outlined;

- Second-Order Design (SOD): the measurement accuracies (weights) are determined;

- Combined-Order Design (COMD): both the Firstand Second-Order Design have to be optimally solved simultaneously; and

- Third-Order Design (THOD): addition of observations to improve an existing network.

The aim of this paper is to solve the CombinedOrder Design (COMD) problem of free geodetic networks in two-dimensional space analytically. In the proposed approach the nonlinear matrix function 
describing the quality of the network are linearized around an initial point using Taylor series. Then using the available optimization methods in operational research, such as nonlinear programming method, the optimum corrections to the initial values are computed.

\section{FREE NETWORK ADJUSTMENT}

The problem of the adjustment of free networks characterized by a singular normal equation matrix was discussed during the last years in a series of publications. For all publications, the Moore-Penrose or the pseudo-inverse of singular normal equation matrix was used (e.g., Bjerhammer, 1967; Mittermayer, 1972). The number of linearly independent estimable parameters is determined by the rank of coefficient matrix within observation equations. The pseudo-inverse has the property of belonging to the class of least-squares solutions with a minimal Euclidean norm of the vector of unknowns. The pseudo-inverse enables the construction of solutions for classically unsolvable adjustment problems.

The generalized inverse of the matrix $\mathrm{N}$ is $\mathrm{N}^{+}$ and can be given by:

$$
\mathbf{N}^{+}=\mathbf{N}(\mathbf{N} \mathbf{N})^{-1}
$$

which achieve the properties:

$$
\begin{aligned}
& \mathbf{N} \mathbf{N}^{+} \mathbf{N}=\mathbf{N} \\
& \mathbf{N}^{+} \mathbf{N} \mathbf{N}^{+}=\mathbf{N}^{+} \\
& \mathbf{N}^{+} \mathbf{N}=\left(\mathbf{N}^{+} \mathbf{N}\right)^{\mathbf{T}} \\
& \mathbf{N} \mathbf{N}^{+}=\left(\mathbf{N ~} \mathbf{N}^{+}\right)^{\mathbf{T}}
\end{aligned}
$$

Thus the cofactor matrix Q can be obtained as:

$$
\mathbf{Q}=\mathbf{N}(\mathbf{N ~ N})^{-1} \mathbf{N}(\mathbf{N ~ N})^{-1} \mathbf{N}
$$

This obtained cofactor matrix $\mathbf{Q}$ achieves the following properties:

$$
\begin{gathered}
\text { 1- It is quadratic, symmetric and singular: } \\
\operatorname{det}(\mathrm{Q})=0
\end{gathered}
$$

2- The solution vector $(x)$ :

$$
\begin{aligned}
& \quad \hat{\mathbf{x}}=\mathbf{Q} \mathbf{U} \\
& \text { where: } \\
& \hat{\mathbf{x}} \text { : has a minimum norm length. } \\
& \qquad \mathbf{x}^{\mathbf{T}} \mathbf{x}=\text { min. }
\end{aligned}
$$$$
\text { 3- } \operatorname{Trace}(Q)=\min \text {. }
$$

The results of free geodetic network adjustment and their superior quality service as an authentic reflection (undistorted mirror image) of the measurements' quality. The above property is particularly important today when we employ GPS measurements for densification of conventional control, where the later is usually of a relatively lower quality (Papo, 1999).

\section{OBJECTIVE FUNCTION FOR COMD PROBLEM OF FREE GEODETIC NETS}

The purpose of this study is to develop a fully analytical mathematical procedure to solve for the optimal improvements to the initial design (initial locations of points and initial weights). The entire solution process can be fully automated by the MATLAB program that was written by the authors without the need for human intervention. In order to solve for the improvements analytically, the major problem is how to bring the quality criteria into a strong mathematical form, i.e., to establish the explicit relation between the preset design criteria of precision, reliability, and cost and the unknown parameters to be optimized, and that is accomplished in this paper by using the technique of Taylor series expansion to linearize the non-linear matrix equations related to network design, converting various network quality requirements into constraints on the unknown parameters to be optimally solved for.

\subsection{Quality Constraints}

The global variance-covariance matrix in the case of free networks is written as:

$$
\mathbf{C}_{\mathbf{x}}=\sigma_{\mathbf{0}}^{2} \mathbf{Q}_{\mathbf{x}}=\sigma_{\mathbf{0}}^{2}\left(\mathbf{A}^{\mathbf{T}} \mathbf{P} \mathbf{A}\right)^{+}
$$

where:

$\sigma_{0}^{2}$ : is the priori variance factor that is usually taken 1.0 at the design stage,

()$^{+}$: representing the reflexive generalized inverse of a matrix.

Note that in two-dimensional space elements of matrix $\mathbf{C}_{\mathbf{x}}$ are nonlinear functions of both the observational weights and station coordinates, that are embedded in the network configuration matrix A. Supplied with initial values of both the coordinates and observation weights, matrix $\mathbf{C}_{\mathbf{x}}$ can be approximated using Taylor series restricted to linear term as follows (Kuang, 1996)

$\mathbf{C}_{\mathbf{x}}=\mathbf{C}_{\mathbf{x}}^{\mathbf{0}}+\sum_{\mathbf{i}=1}^{\mathbf{m}} \frac{\partial \mathbf{C}_{\mathbf{x}}}{\partial \mathbf{x}_{\mathbf{i}}} \Delta \mathbf{x}_{\mathbf{i}}+\sum_{\mathbf{i}=1}^{\mathbf{m}} \frac{\partial \mathbf{C}_{\mathbf{x}}}{\partial \mathbf{y}_{\mathbf{i}}} \Delta \mathbf{y}_{\mathbf{i}}+\sum_{\mathbf{i}=1}^{\mathbf{m}} \frac{\partial \mathbf{C}_{\mathbf{x}}}{\partial \mathbf{p}_{\mathbf{i}}} \Delta \mathbf{p}_{\mathbf{i}}$

where:

$$
\mathbf{C}_{\mathbf{X}}^{\mathbf{0}}=\sigma_{\mathbf{0}}^{2}\left\{\left(\mathbf{A}^{\mathbf{T}} \mathbf{P} \mathbf{A}\right)^{+}\right\}_{\mathbf{x}^{0}, \mathbf{y}^{\mathbf{0}}, \mathbf{z}^{\mathbf{0}}, \mathbf{p}^{\mathbf{0}}}
$$




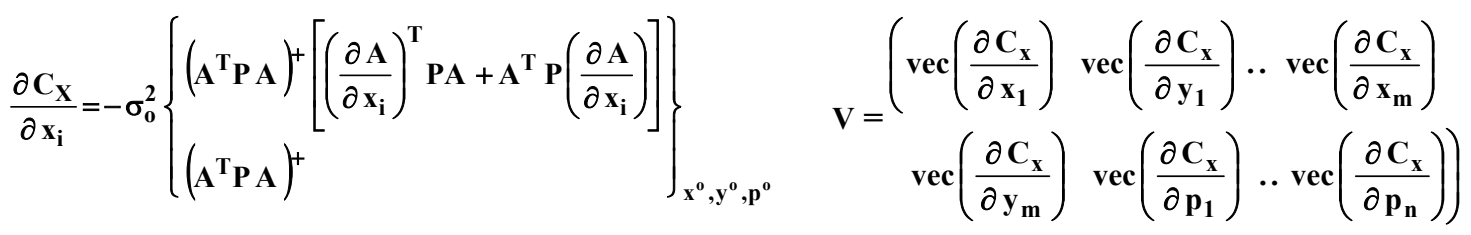

$$
\begin{aligned}
& \mathbf{w}=\left(\Delta \mathbf{x}_{1}, \Delta \mathbf{y}_{1}, \ldots \Delta \mathbf{x}_{m}, \Delta \mathbf{y}_{m}, \Delta p_{1} \ldots \Delta p_{n}\right)^{T} \\
& V_{1}=\left(\begin{array}{lll}
I_{u} \Theta & I_{u}
\end{array}\right)^{T} \mathbf{V}, \quad \mathbf{u}_{1}=\left(I_{\mathbf{u}} \Theta I_{\mathbf{u}}\right)^{\mathrm{T}} \mathbf{u}
\end{aligned}
$$$$
\frac{\partial \mathbf{C}_{\mathbf{X}}}{\partial \mathbf{y}_{\mathbf{i}}}=-\sigma_{\mathbf{0}}^{2}\left\{\begin{array}{l}
\left(\mathbf{A}^{\mathbf{T}} \mathbf{P} \mathbf{A}\right)^{+}\left[\left(\frac{\partial \mathbf{A}}{\partial \mathbf{y}_{\mathbf{i}}}\right)^{\mathbf{T}} \mathbf{P A}+\mathbf{A}^{\mathbf{T}} \mathbf{P}\left(\frac{\partial \mathbf{A}}{\partial \mathbf{y}_{\mathbf{i}}}\right)\right] \\
\left(\mathbf{A}^{\mathbf{T}} \mathbf{P} \mathbf{A}\right)^{+}
\end{array}\right\}_{\mathbf{x}^{0}, \mathbf{y}^{0}, \mathbf{p}^{\mathbf{0}}}
$$$$
\frac{\partial \mathbf{C}_{\mathbf{X}}}{\partial \mathbf{p}_{\mathbf{i}}}=-\sigma_{\mathbf{0}}^{2}\left\{\left(\mathbf{A}^{\mathbf{T}} \mathbf{P} \mathbf{A}\right)+\left[\mathbf{A}^{\mathbf{T}}\left(\frac{\partial \mathbf{P}}{\partial \mathbf{p}_{\mathbf{i}}}\right) \mathbf{A}\right]\left(\mathbf{A}^{\mathbf{T}} \mathbf{P} \mathbf{A}\right)^{+}\right\}_{\mathbf{x}^{0}, \mathbf{y}^{0}, \mathbf{p}^{\mathbf{0}}}
$$

$\boldsymbol{\Theta}$ : represents the Khatri-Rao product, which for any two arbitrary matrices $B_{k, m}$ and $A_{n, m}$ (with the same number of columns) yields a matrix $C_{k \times n, m}$ (Doma, 2004).

\subsection{Physical Constraints}

Also, the position improvement to be introduced should be bounded by the topography consideration that may be established in the field by reconnaissance i.e.;

$$
\begin{aligned}
& a_{1 i} \leq \Delta x_{i} \leq a_{2 i} \\
& b_{1 i} \leq \Delta y_{i} \leq b_{2 i} \quad(i=1,2, \ldots ., m)
\end{aligned}
$$

Usually, criterion matrix $\mathrm{C}_{S}$ is used as the precision criteria in this case; the design problem seeks an optimal configuration matrix A and weight matrix $\mathrm{P}$ such that $\mathrm{C}_{\mathrm{S}}$ can be best approximated by $\mathrm{C}_{\mathrm{x}}$. The precision function in this case is:

$$
\left\|C_{x}-C_{S}\right\|=\min \quad \text { (optimal design) }
$$
where:

$$
\|\| \text { : Represents norm of the matrix. }
$$

In order to avoid the precision of some of the coordinates that becomes disproportionally better than the others. Schaffrin, 1981 adds another expression for this criterion so:

$$
\operatorname{vec}\left(C_{x}\right) \leq \operatorname{vec}\left(C_{S}\right) \quad \text { (precision control) }
$$

where:

vec: The operation produces a vector by staking the column of a quadratic matrix under another in a single column.

Finally, by linearization of the precision criteria, we can reformulate precision criteria in a compact matrix and vector form:

$$
\begin{array}{rr}
\|V \cdot w-u\|=\min & \text { (optimal design) } \\
V_{1} \cdot w-u_{1} \leq 0 & \text { (precision control) }
\end{array}
$$

Denote:

$$
\mathbf{u}=\operatorname{vec}\left(C_{S}\right)-\operatorname{vec}\left(C_{x}^{0}\right)
$$

the above equation can be written as

$$
\mathbf{l b} \leq \mathbf{w} \leq \mathbf{u b}
$$

where: $\mathbf{l b}$ and $\mathbf{u b}$ : lower and upper bounds of unknowns

\subsection{Evalutaion Of Differentials}

It is noticeable that before starting the formulation of mathematical model for optimization model, we need also partial derivative of configuration matrix $\mathrm{A}$, and weight matrix $\mathrm{P}$ with respect to coordinates of network depending on types of observations (Kuang, 1996).

The elements of the configuration matrix A are formed as linear or nonlinear functions of the approximate coordinates. The form of functions that constitute the elements of the configuration matrix A can be determined geometrically depending on the specific types of observables proposed. Matrix $\mathrm{P}$ contains the individual observation weights of the observables (more details in Doma 2004).

\section{FORMULATION OF THE MATHEMAT- ICAL MODEL FOR COMD PROBLEM}

In the summary, optimization means that determination of maximum or minimum of one target function under some conditions. For example, in the geodetic network the target function will be on which represents the network quality i.e. precision, reliability, and cost. 
This objective function should be designed in such a way that (Kiamehr, 2003):

- It must realize the required network quality i.e. precision, reliability, and cost of network and deformation parameters.

- Resistance to gross error in observations and minimize the effects of undetected gross errors.

- It can allow testing of hypothesis with higher significance.

In the present study, a suitable target function that includes precision requirements and physical constraints (topography) has been applied. This method analytically performs the combined First and Second Order Design (Vanicek and Krakiwsky 1986) i.e. the simultaneous optimization of the geometrical configuration and weight of observation in any positioning or monitoring network. In fact, the position of net points selected in reconnaissance cannot be changed, the mathematical model can be reduced to the Second Order Design. On the other hand, if we take the accuracy of measurements as fixed, the model reduces to the First Order Design. One may formulate the mathematical model for optimization as follows:

$$
\begin{aligned}
& \text { Minimize: }\|\mathbf{V} \cdot \mathbf{w}-\mathbf{u}\| \\
& \text { subjected to : } \\
& \qquad \mathbf{V}_{\mathbf{1}} \cdot \mathbf{w}-\mathbf{u}_{\mathbf{1}} \leq \mathbf{0} \\
& \mathbf{l b} \leq \mathbf{w} \leq \mathbf{u b}
\end{aligned}
$$

where:

$\mathrm{lb}$ and ub : lower and upper

\section{APPLICATION EXAMPLE}

To show the efficiency of using the proposed approach for optimization, an example is given in the current study. This example illustrates the application of the proposed approach to the optimal design of a free geodetic network in two-dimensional space. The above-mentioned model is a single object optimization problem and for solving it one can use the mathematical programming method (linear or nonlinear programming method). Using the PcMatlab version with a personal computer and the developed mathematical model, a computer program has been designed and written by authors to solve the above mathematical model. This program is based on the variation of coordinate method and the variancecovariance matrix. A two-dimensional trilateration network comprising ten points (20 coordinates) is considered. In this trilateratin network, distances are observed (Figure, 1). The simulated approximate coordinates are listed in Table (1). It is assumed that an EDM instrument will be used to measure all the distances with an achievable accuracy $\sigma_{\mathrm{s}}^{2}=(0.5 \mathrm{~mm})^{2}+(1 \mathrm{ppm} . \mathrm{S})^{2}$, where S is the distance computed from the approximate coordinates. The optimization is done under precision criteria; all the standard deviations of the coordinate components must be less than $1.0 \mathrm{~mm}$.

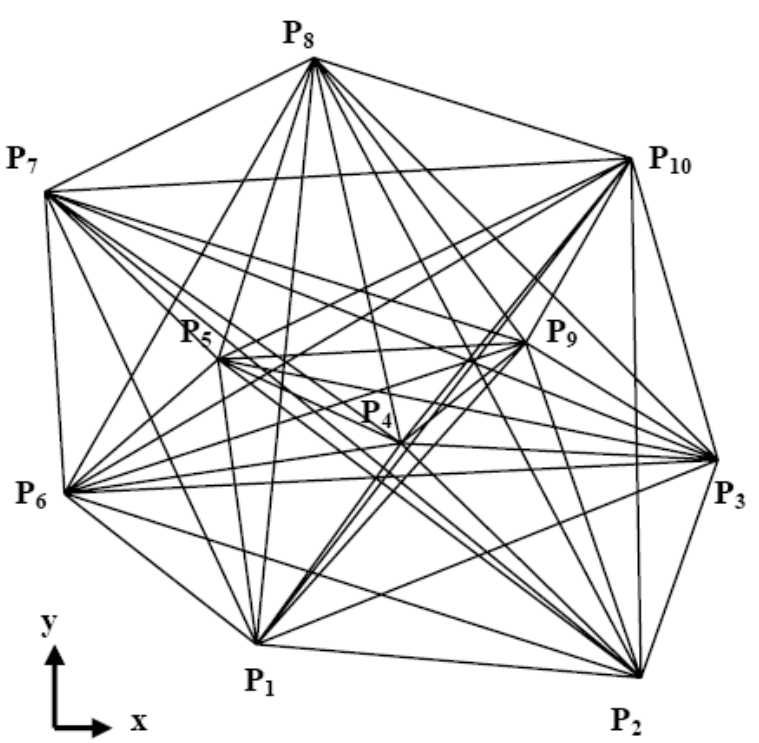

Figure (1): The Two-Dimensional Free Trilateration Network

Table (1): The simulated approximate coordinates of network points and the initial standard deviations of new pints

\begin{tabular}{|c|c:c|c:c||}
\hline \multirow{3}{*}{ Points } & \multicolumn{2}{|c|}{$\begin{array}{c}\text { Simulated } \\
\text { coordinates } \\
\text { of netpoints }\end{array}$} & \multicolumn{2}{|c|}{$\begin{array}{c}\text { Initial standard } \\
\text { deviations }\end{array}$} \\
\cline { 2 - 5 } & $\begin{array}{c}\mathbf{x} \\
\mathbf{~ m})\end{array}$ & $\begin{array}{c}\mathbf{y} \\
\mathbf{~ m})\end{array}$ & $\begin{array}{c}\boldsymbol{\sigma}_{\mathbf{x}} \\
(\mathbf{m m})\end{array}$ & $\begin{array}{c}\mathbf{\sigma}_{\mathbf{y}} \\
(\mathbf{m m})\end{array}$ \\
\hline $\mathbf{P}_{\mathbf{1}}$ & 2000 & 1000 & 1.59 & 1.44 \\
\hline $\mathbf{P}_{\mathbf{2}}$ & 5000 & 100 & 2.00 & 1.72 \\
\hline $\mathbf{P}_{\mathbf{3}}$ & 5400 & 2600 & 1.61 & 1.55 \\
\hline $\mathbf{P}_{\mathbf{4}}$ & 3000 & 3000 & 1.45 & 1.27 \\
\hline $\mathbf{P}_{\mathbf{5}}$ & 1800 & 4600 & 1.49 & 1.23 \\
\hline $\mathbf{P}_{\mathbf{6}}$ & 400 & 2600 & 1.52 & 1.48 \\
\hline $\mathbf{P}_{\mathbf{7}}$ & 200 & 6000 & 1.42 & 1.65 \\
\hline $\mathbf{P}_{\mathbf{8}}$ & 2000 & 7000 & 1.46 & 1.45 \\
\hline $\mathbf{P}_{\mathbf{9}}$ & 4000 & 5000 & 1.54 & 1.32 \\
\hline $\mathbf{P}_{\mathbf{1 0}}$ & 5000 & 7000 & 1.85 & 1.86 \\
\hline
\end{tabular}

We would like to find optimal configuration (coordinates shifts) and observational plan (weight of observations) in this criterion. In the present case study, we assume that topographical constraints for shifting of coordinates are in the range from -50 to $50 \mathrm{~m}$ in $\mathrm{x}-$, $\mathrm{y}$-directions. 


\section{RESULTS AND DISCUSSIONS}

In this case, both the network configuration and the observational plan have to be optimally determined in order to achieve the above set precision criteria. The optimization algorithm starts with an initial design that proposes both the approximate locations of network stations as well as the types and precision of observations to be made. In this case study, we assume that the network stations have been chosen as shown in Figure (1). As for the observing plan, the initial weights needed to initialize the optimization process have been listed in Table (3) for all the proposed observations.

The obtained optimization results are listed in Tables (2) to (4) and in Figure (2) and (3).

Table (2) gives the optimal coordinate shifts solved for each point by optimization model, and the optimal station coordinates.

Table (2): Coordinate shifts and optimal design of the network configuration

\begin{tabular}{|c|c|c|c|c|}
\hline \multirow[t]{2}{*}{ Point } & \multicolumn{2}{|c|}{$\begin{array}{c}\text { Optimal } \\
\text { coordinate shifts }\end{array}$} & \multicolumn{2}{|c|}{$\begin{array}{c}\text { Final coordinate of } \\
\text { netpoints }\end{array}$} \\
\hline & $\Delta x(m)$ & $\Delta y(m)$ & $\mathbf{x}(\mathrm{m})$ & $y(\mathbf{m})$ \\
\hline $\mathbf{P}_{1}$ & -50 & -50 & 1950 & 950 \\
\hline $\mathbf{P}_{2}$ & -50 & 50 & 4950 & 150 \\
\hline $\mathbf{P}_{3}$ & 50 & 50 & 5450 & 2650 \\
\hline $\mathbf{P}_{4}$ & 50 & -50 & 3050 & 2950 \\
\hline $\mathbf{P}_{5}$ & -50 & 50 & 1750 & 4649.622 \\
\hline $\mathbf{P}_{6}$ & -50 & -50 & 350 & 2550 \\
\hline $\mathbf{P}_{7}$ & 5.34 & -50 & 205.34 & 5950 \\
\hline $\mathbf{P}_{8}$ & 50 & 39.01 & 2050 & 7039.01 \\
\hline $\mathbf{P}_{9}$ & 50 & 40.55 & 4050 & 5040.55 \\
\hline $\mathbf{P}_{10}$ & -50 & -50 & 4950 & 6950 \\
\hline
\end{tabular}

Figure (2) shows the initial and optimal locations of the netpoints. To clarify the shift (displacement) for each point of the network, Figure (3) shows the direction and value of shift for each point of network with magnification ratio $=10$.

Table (3) lists the initial weights, optimal weights and the repetition numbers. From Table (3), one can see that, all the observations have positive weights and these weights are bigger than an initial weights, this means that there are no deleted observations in the final observational plane and in order to achieve the standard deviation of $1.0 \mathrm{~mm}$ for coordinate components, the repetition numbers of the observations calculated from the optimal weights.

Table (4), gives a comparison between the required standard deviations of the coordinate components and the actual achieved. From the obtained results, it can be found that:
1. The coordinate shifts for each point after applying the proposed mathematical model are ranged from $50 \mathrm{~m}$ at point $\mathrm{P}_{2}$ to $5.34 \mathrm{~m}$ at point $\mathrm{P}_{7}$. These results show clearly that all the final shifts of the coordinate components are in the range of the choosen topographical constraint.

2- The standard deviations of the netpoints after applying the optimization model are ranged from $1.0 \mathrm{~mm}$ at point $\mathrm{P}_{4}$ to $0.82 \mathrm{~mm}$ at point $\mathrm{P}_{3}$. So, all standard deviations of the coordinate components are less than and close to the required value $(1 \mathrm{~mm})$.

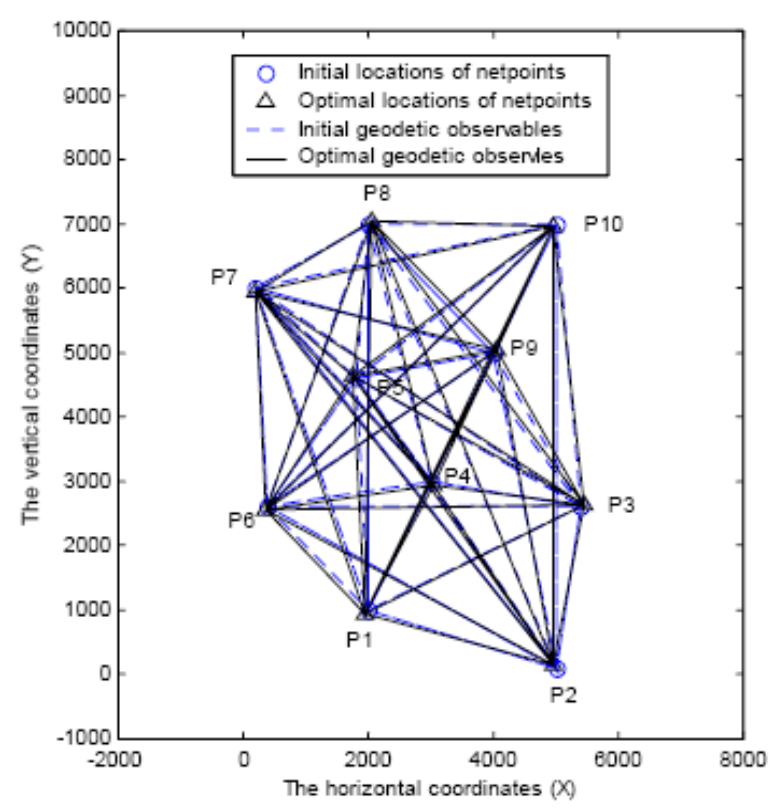

Figure (2): The initial and the optimal locations of netpoints

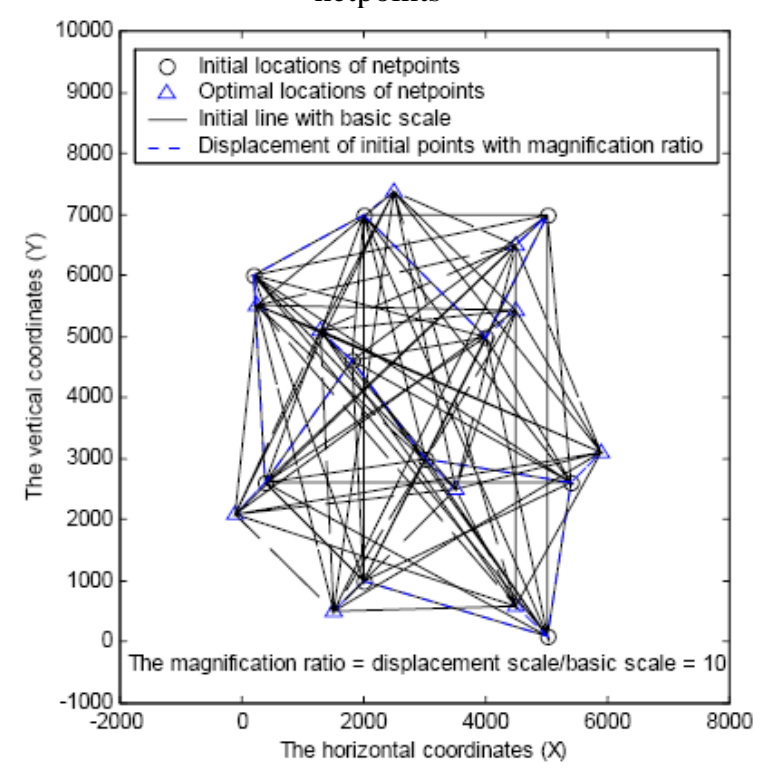

Figure (3): Initial locations of netpoints and their displacement with magnification ratio 
Table (3): The initial weights vector, optimal weights vector and repetition no.

\begin{tabular}{|c|c|c|c|c|c|}
\hline \multirow[t]{2}{*}{ So. } & \multicolumn{2}{|c|}{$\begin{array}{c}\text { Observation } \\
\text { lines }\end{array}$} & \multirow{2}{*}{$\begin{array}{c}\text { Initial } \\
\text { weights } \\
\mathbf{P}_{\text {(Initial) }}\end{array}$} & \multicolumn{2}{|c|}{ Optimal design } \\
\hline & From & To & & $\mathbf{P}_{\text {(Optimal) }}$ & $\begin{array}{c}\text { Repetition } \\
\text { no. }\end{array}$ \\
\hline 1 & 1 & 2 & 0.1019 & 0.3399 & 4 \\
\hline 2 & 1 & 3 & 0.0708 & 0.3060 & 5 \\
\hline 3 & 1 & 4 & 0.2000 & 0.2505 & 2 \\
\hline 4 & 1 & 5 & 0.0769 & 0.1542 & 3 \\
\hline 5 & 1 & 6 & 0.1953 & 0.3216 & 2 \\
\hline 6 & 1 & 7 & 0.0354 & 0.3065 & 9 \\
\hline 7 & 1 & 8 & 0.0278 & 0.1170 & 5 \\
\hline 8 & 1 & 9 & 0.0500 & 0.2253 & 5 \\
\hline 9 & 1 & 10 & 0.0222 & 0.0740 & 4 \\
\hline 10 & 2 & 3 & 0.1560 & 0.4225 & 3 \\
\hline 11 & 2 & 4 & 0.0806 & 0.1522 & 2 \\
\hline 12 & 2 & 5 & 0.0328 & 0.2113 & 7 \\
\hline 13 & 2 & 6 & 0.0365 & 0.2886 & 8 \\
\hline 14 & 2 & 7 & 0.0173 & 0.0346 & 3 \\
\hline 15 & 2 & 8 & 0.0177 & 0.0216 & 2 \\
\hline 16 & 2 & 9 & 0.0400 & 0.1599 & 4 \\
\hline 17 & 2 & 10 & 0.0210 & 0.2263 & 11 \\
\hline 18 & 3 & 4 & 0.1689 & 0.2413 & 2 \\
\hline 19 & 3 & 5 & 0.0590 & 0.2112 & 4 \\
\hline 20 & 3 & 6 & 0.0400 & 0.1662 & 5 \\
\hline 21 & 3 & 7 & 0.0259 & 0.0864 & 4 \\
\hline 22 & 3 & 8 & 0.0323 & 0.2627 & 9 \\
\hline 23 & 3 & 9 & 0.1295 & 0.2423 & 2 \\
\hline 24 & 3 & 10 & 0.0512 & 0.2236 & 5 \\
\hline 25 & 4 & 5 & 0.2500 & 0.2500 & 1 \\
\hline 26 & 4 & 6 & 0.1445 & 0.3109 & 3 \\
\hline 27 & 4 & 7 & 0.0594 & 0.1803 & 4 \\
\hline 28 & 4 & 8 & 0.0588 & 0.1424 & 3 \\
\hline 29 & 4 & 9 & 0.2000 & 0.2242 & 2 \\
\hline 30 & 4 & 10 & 0.0500 & 0.2296 & 5 \\
\hline 31 & 5 & 6 & 0.1678 & 0.2369 & 2 \\
\hline 32 & 5 & 7 & 0.2212 & 0.2322 & 2 \\
\hline 33 & 5 & 8 & 0.1724 & 0.2210 & 2 \\
\hline 34 & 5 & 9 & 0.2000 & 0.2693 & 2 \\
\hline 35 & 5 & 10 & 0.0625 & 0.3199 & 6 \\
\hline 36 & 6 & 7 & 0.0862 & 0.2997 & 4 \\
\hline 37 & 6 & 8 & 0.0456 & 0.2826 & 7 \\
\hline 38 & 6 & 9 & 0.0534 & 0.2126 & 4 \\
\hline 39 & 6 & 10 & 0.0247 & 0.0311 & 2 \\
\hline 40 & 7 & 8 & 0.2358 & 0.3626 & 2 \\
\hline 41 & 7 & 9 & 0.0648 & 0.3168 & 5 \\
\hline 42 & 7 & 10 & 0.0416 & 0.2469 & 6 \\
\hline 43 & 8 & 9 & 0.1250 & 0.2848 & 3 \\
\hline 44 & 8 & 10 & 0.1111 & 0.3347 & 4 \\
\hline 45 & 9 & 10 & 0.2000 & 0.2198 & 2 \\
\hline
\end{tabular}

Table (4): Comparison between the required and achievable precisions of the coordinate components after optimization.

\begin{tabular}{|l|c:c|c:c|}
\hline \multirow{2}{*}{ Point } & \multicolumn{2}{|c|}{$\begin{array}{c}\text { Required } \\
\text { precision }\end{array}$} & \multicolumn{2}{c|}{$\begin{array}{c}\text { Achievable } \\
\text { precision }\end{array}$} \\
\cline { 2 - 5 } & $\begin{array}{c}\boldsymbol{\sigma}_{\mathbf{x}} \\
(\mathbf{m m})\end{array}$ & $\begin{array}{c}\boldsymbol{\sigma}_{\mathbf{y}} \\
(\mathbf{m m})\end{array}$ & $\begin{array}{c}\boldsymbol{\sigma}_{\mathbf{x}} \\
(\mathbf{m m})\end{array}$ & $\begin{array}{c}\boldsymbol{\sigma}_{\mathbf{y}} \\
(\mathbf{m m})\end{array}$ \\
\hline $\mathbf{P}_{\mathbf{1}}$ & 1.00 & 1.00 & 0.89 & 0.84 \\
\hline $\mathbf{P}_{\mathbf{2}}$ & 1.00 & 1.00 & 0.97 & 0.87 \\
\hline $\mathbf{P}_{\mathbf{3}}$ & 1.00 & 1.00 & 0.91 & 0.82 \\
\hline $\mathbf{P}_{\mathbf{4}}$ & 1.00 & 1.00 & 1.00 & 0.91 \\
\hline $\mathbf{P}_{\mathbf{5}}$ & 1.00 & 1.00 & 0.95 & 0.88 \\
\hline $\mathbf{P}_{\mathbf{6}}$ & 1.00 & 1.00 & 0.87 & 0.84 \\
\hline $\mathbf{P}_{\mathbf{7}}$ & 1.00 & 1.00 & 0.84 & 0.90 \\
\hline $\mathbf{P}_{\mathbf{8}}$ & 1.00 & 1.00 & 0.87 & 0.87 \\
\hline $\mathbf{P}_{\mathbf{9}}$ & 1.00 & 1.00 & 0.92 & 0.87 \\
\hline $\mathbf{P}_{\mathbf{1 0}}$ & 1.00 & 1.00 & 0.92 & 0.93 \\
\hline
\end{tabular}

\section{CONCLUSION}

Free geodetic networks are still very popular in geodesy, surveying and mapping mainly due to their unique property of being independent of errors in external data.

In this paper, the presented mathematical model deals with the simultaneous optimization of the network configuration (FOD Problem) and observational weights (SOD Problem), this problem is known as COMD Problem. For practical applications, after appropriate network quality criteria are given, this optimization procedure gives the optimal weights or standard deviations for each observable as well as the optimal position improvements of the initially selected points to obtain an optimal configuration of the network. Therefore, the optimization procedure developed is very flexible and practical. It is preferable to use the proposed mathematical model instead of the trial and error method.

\section{REFERENCES}

Amiri Seemkooei, A. (2001). "Comparison of reliability and geometrical strength criteria in geodetic networks.” Journal of Geodesy (2001), 75: 227-233.

Bjerhammer, A. (1967). "Studies with Genralized Matrix Algebra.” Bull. Geodesique, no. 85.

Cross, P.A.(1985). "Numerical methods in network design". In: "Optimization and design of geodetic networks.” Edited by E. Grafarend and F. Sanso, Spring-Verlag, Berlin.

Doma, M.I. (2004). “Application of Optimization Theory to Geodetic Networks Using Different Orders Design Problems” M. Sc. 
Thesis, Faculty of Engineering, Minufiya University, Minufiya, Egypt.

Kiamehr, R., (2003). "Multi Object Optimization of Geodetic Network" NCC Geomatics 82 conferences, Tehran, Iran. Accepted NCC J. Engineering Surveying.

Kuang, S.L. (1996).”Geodetic Network Analysis and Optimal Design: Concept and Applications." Ann Arbor Press, Inc., Chelsea, Michigan.

Papo, H. (1999). "Datum accuracy and its dependence on network geometry" International Scientific and Technical Conference dedicated to the $220^{\text {th }}$ anniversary of Moscow State University of Geodesy and Cartography, May 24-29, 1999.

Schaffrin, B. (1981): "Some proposals concerning the diagonal second order design of geodetic networks.” Manuscripta Geodetica, 6 (3), 303-326.
Schmitt, G.(1982). "Optimization of geodetic networks” Review of Geophysics and Space Physics, Volume 20, No.4.

M. Onur Kaplan; Tevfik Ayan, and Serdar Erol, 2004. "The Effects of Geodetic Configuration of the Network in Deformation Analysis" FIG Working Week 2004, Athens, Greece, May 22-27, 2004.

Mittermayer, E. (1972). “A Generalization of the Least-Squares Method for the Adjustment of Free Networks.” Bulletin Geodesique, No. 104.

Rahil, A.M.F. (2005). "A new proposed for solving the combined order design problem" ERB, Vol. 28, No. 3, 2005, Menufiya University, Faculty of Engineering, Shebien El-Koom, Egypt, ISSN 1110-1180.

Vanicek, P. and E.J. Krakiwsky (1986). "Geodesy the concepts. Second edition” North-Holland Publishing company. 